P.027

\section{New adrenergic baroreflex evaluation in Valsalva maneuver}

IS Palamarchuk (London)* J Baker (London) K Kimpinski (London)

doi: $10.1017 /$ cjn.2015.138

Background: Valsalva maneuver (VM) is a simple and noninvasive technique extensively utilized clinically to detect dysautonomia. VM provides detailed information of baroreflex sensitivity (BRS) which is an important cardiovascular and autonomic marker. However, the current approach for calculating its adrenergic component (BRSa1) is moderately reliable and fails to evaluate atypical VM patterns. Methods: We analyzed typical and atypical VM patterns of 89 young, healthy individuals $(30 \pm 13$ years $)$ with the aim of improving BRSa evaluation. Objectives: 1) To determine a new BRSa calculation (BRSa2) applicable to different VM patterns; 2) correlate $\mathrm{BRSa} 2$ to $\mathrm{BRSa} 1 ; 3$ ) compare the internal consistency (ICC) between BRSa1 and BRSa2. Results: The BRSa2 calculation is a complex hemodynamic and time assessment equivalent to the slope in vagal BRS. In contrast to BRSa1, BRSa2 operates with hemodynamic indices easily detectable in any VM pattern. In atypical VM patterns, BRSa2 correlated with BRSa1: "flat-top responses" $(r=0.774, p<0.01)$; rapid hemodynamic recovery $(r=0.461, p$ $<0.05)$. Most importantly, BRSa2 was more reliable than BRSa1 ( $\mathrm{ICC}=0.759$ versus 0.469 ). Conclusion: $\mathrm{BRSa} 2$ is more reliable and allows atypical responses to VM to be analyzed, which clinically, could help differentiate natural physiological variances and mild adrenergic dysfunction.

\section{P.028}

\section{Severe necrotizing myelopathy from toxacariasis}

R Magun (Hamilton)* JE Silva (Hamilton)

doi: $10.1017 / \operatorname{cjn} .2015 .139$

We present a 25 year old female veterinarian technician presenting with rapidly progressive quadriplegia in less then 12 hours. Her symptoms occurred at work with initially bilateral hand weakness followed by arm and leg weakness. Before the end of the day she was on a ventilator in the ICU. MRI showed a hyperintense longitudinal T2 signal extending from the cervical medullary junction to T1. Extensive cervical spinal cord edema with cord expansion was noted. CSF showed normal protein and cell count with no oligoclonal banding. A post-infectious inflammatory process causing transverse myelitis was presumed and she was given IVIG, steroids, and plasmapheresis with no improvement. A serum ELISA test for IgG to Toxocara was reactive at titre of 1:800 at 3 weeks after her initial presentation. Her serum IgE levels was elevated at $169 \times 10 \times 3 \mathrm{U} / \mathrm{L}$ (Normal $<87 \mathrm{x}$ $10 \times 3 \mathrm{U} / \mathrm{L})$. At 4 weeks, she was commenced on albendazole at 800 mg per day for two months. A repeat serum ELISA test at 6 weeks and 2 weeks into her treatment with albendazole showed a declining titre of 1:200 consistent with recent Toxocara infection. At 10 weeks, her ELISA test was non-reactive. Unfortunately she did not respond to albendazole treatment and she shows minimal improvement now 1.5 years later.

\section{P.029}

\section{Cryptococcoma in Idiopathic CD4 Lymphopenia}

$S$ Alshimemeri (vancouver)* G Walker (vancouver) M Mezei (vancouver) $S$ Warner (vancouver) L Viana (vancouver) JJ Barton (vancouver)

doi: $10.1017 /$ cjn.2015.140

Idiopathic CD4 lymphopenia is a rare immune disorder that renders patients vulnerable to unusual infections. A 25-year-old female had been diagnosed with idiopathic CD4 lymphopenia (CD4 count of 50/uL) at age 14 after pulmonary infection with atypical mycobacterium, but had since been asymptomatic on Trimethoprim/ Sulfamethoxazole prophylaxis. She presented now with 5 weeks of headache, vomiting, diplopia, and vertigo. This had been diagnosed as benign positional vertigo. However, neuro-ophthalmologic exam revealed gaze-evoked nystagmus, impaired smooth pursuit, a left hypertropic skew deviation, left sensorineural hearing loss, gait ataxia and left limb dysmetria. MRI brain showed a $15 \mathrm{~mm}$ extra-axial enhancing mass at the left cerebellopontine angle, and chest $\mathrm{CT}$ showed pulmonary lesions. CSF Cryptococcal antigen was highly positive $(>1: 1024)$ and CSF culture grew Cryptococcus neoformans variety grubii. She was treated with amphotericin B $175 \mathrm{mg}$ daily and 5-Flucytosine $1000 \mathrm{mg}$ QID for 4 weeks, followed by Fluconazole $400 \mathrm{mg}$ daily, and made an excellent recovery. Cryptococcal infection usually presents as a meningitis, but can occasionally present as a mass lesion. Cryptococcal infection is one of the most common complications in idiopathic CD4 lymphopenia. This case illustrates the importance of neurologic signs in correctly localizing the lesion and the need for high suspicion of serious pathology in patients with rare immune disorders.

\section{P.030}

Ramsay Hunt Syndrome associated with central nervous system involvement in an adult: a case report

\section{Chan (London)* A Cartagena (London) SM Hosseini (London) A Bombassaro (London)}

doi: 10.1017/cjn.2015.141

Background: Ramsay Hunt Syndrome with cerebellar encephalitis is rare in adult. Case Report: We describe a 55-year-old diabetic female who presented with truncal ataxia, right peripheral facial palsy and right ear pain associated with erythematous vesicular lesions in her external auditory canal. Later, she developed dysmetria, fluctuating diplopia and dysarthria. No facial lesions were identified and lesions were limited to the external auditory canal. Cerebral spinal fluid tested positive for varicella zoster virus polymerase chain reaction. She was diagnosed with Ramsay Hunt Syndrome with spread to the central nervous system and treated with acyclovir intravenous therapy (10 mg/kg every 8 hours). Her facial palsy completely resolved within 48 hours of acyclovir treatment, however, vesicular lesions, imbalance and cerebellar symptoms remained; a tapering course of high dose prednisone was then added.

Discussion: Prognosis for facial palsy is poor in Ramsay Hunt Syndrome: Only $10 \%$ of patients will have complete resolution of their facial palsy. Improvement of facial palsy may be a good marker for response to treatment. Conclusion: Varicella zoster virus reactivation affecting the central nervous system in adults is rare. Knowledge 